\title{
Poliolefinas Reforçadas com Fibras Vegetais Curtas: Sisal vs. Curauá
}

\author{
Márcia A. S. Spinacé \\ Centro de Ciências Naturais e Humanas, UFABC \\ Lea G. Janeiro, Filippe C. Bernardino, Thais A. Grossi, Marco-A. De Paoli \\ Laboratório de Polímeros Condutores e Reciclagem, UNICAMP
}

Resumo: É crescente o interesse nos compósitos poliméricos reforçados com fibras vegetais curtas em substituição às fibras de vidro, pois as fibras naturais provêm de fontes renováveis, não são abrasivas aos equipamentos de processamento, são biodegradáveis, e possuem baixa densidade comparada às fibras de vidro. Elas apresentam início de degradação em torno de $200{ }^{\circ} \mathrm{C}$, sendo adequadas para reforçar poliolefinas que são processadas até essa temperatura ou termofíxos. Várias fibras vegetais vêm sendo usadas como reforço, dentre elas o curauá e o sisal; no entanto, há grande controvérsia na literatura sobre as propriedades finais dos compósitos. Neste trabalho comparamos as propriedades de compósitos de polietileno de alta densidade ou polipropileno com $20 \%$ em massa de fibras curtas de sisal ou de curauá com ou sem agentes de acoplagem. Todos foram processados por extrusão e moldados por injeção, exatamente nas mesmas condições, e os resultados foram comparados em termos das propriedades mecânicas. As fibras de curauá apresentam resistência à tração superior às fibras de sisal e os compósitos com fibras de curauá apresentaram resistência à tração e flexão ligeiramente superior aos compósitos com fibra de sisal. No caso da resistência ao impacto a situação se inverte. Como o sisal é mais frágil que o curauá, durante o processamento ocorre maior quebra da fibra provocando essa diferenciação nas propriedades mecânicas dos compósitos.

Palavras-chave: Fibras vegetais, curauá, sisal, compósito polimérico, extrusão.

\section{Polyolefins Reinforced with Short Vegetal Fibers: Sisal vs. Curauá}

\begin{abstract}
There is growing interest in reinforced polymer composites using short vegetal fibers to replace glass fibers for several reasons. The composite fibers are produced from renewable resources, being biodegradable and less abrasive to the processing equipment, in addition to possessing a lower density than the glass fibers. Since their thermal degradation onset is at $200{ }^{\circ} \mathrm{C}$, they can be used to reinforce thermoplastics processed below this temperature and thermosets. Several vegetal fibers have been used as reinforcing agent, including sisal and cuaruá. However, there is controversy in the literature about the composites final properties. In this work we compare the properties of composites of high density polyethylene or polypropylene with $20 \mathrm{wt}$. (\%) of short sisal or curauá fibers, with or without a coupling agent. All composites were processed by extrusion and molded by injection, under exactly the same conditions, and the mechanical properties were compared. The curauá fibers presented a higher tensile resistance than the sisal fibers, and the composites with curauá fibers had slightly higher tensile and flexural resistance compared to the sisal fiber composites. The situation is opposite in the impact resistance results, with sisal composites displaying higher impact resistance. Since sisal fibers are more fragile than curauá fibers, during processing there is a higher fracture of sisal in comparison to curauá, inducing these differences in composites mechanical properties.
\end{abstract}

Keywords: Vegetal fibers, curauá, sisal, polymeric composite, extrusion.

\section{Introdução}

O consumo de polímeros no Brasil é crescente e, segundo a Associação Brasileira da Indústria do Plástico (Abiplast), em 2008 o consumo brasileiro de termoplásticos foi de $5,3 \times 10^{6} t^{[1]}$. Em 2008, os polímeros mais produzidos foram o polipropileno (PP, 25\%), o policloreto de vinila (PVC, 16\%), o polietileno de alta densidade (PEAD, 15\%), o polietileno de baixa densidade (PEBD, 12\%), o polietileno de baixa densidade linear (PEBDL, 12\%), o poli(tereftalato de etileno) (PET, 12\%) e o poliestireno (PS, 7\%) ${ }^{[1]}$. As poliolefinas são usadas como matéria-prima para muitos setores industriais, tanto na forma pura como reforçadas com fibras curtas de vidro (SFRP- "short fibers reinforced plastics") visando aumentar a resistência mecânica para aplicações estruturais.

Este material reforçado, SFRP, também chamado de compósito polimérico se constitui de uma fase contínua (matriz polimérica) e uma fase dispersa. Essa fase dispersa, mais resistente que a matriz polimérica, pode ser talco ou fibras curtas de vidro. A fibra de vidro é abrasiva aos equipamentos de processamento, não é biodegradável, sua produção demanda um alto consumo de energia e tem alta densidade $\left(2500 \mathrm{~kg} \cdot \mathrm{m}^{-3}\right)$. A utilização das fibras vegetais (com densidades da ordem de 1100 a $1500 \mathrm{~kg} . \mathrm{m}^{-3}$ ) como reforço de polímeros, em substituição das fibras de vidro, tem sido impulsionada tanto pela necessidade de usar matérias primas de fontes renováveis, como pelas vantagens que o uso das fibras vegetais pode ter. No entanto, as fibras vegetais ou lignocelulósicas apresentam início de degradação a ca. de 200-220 ${ }^{\circ} \mathrm{C}$, sendo adequadas somente para reforçar polímeros que sejam processados até essa temperatura ou termofíxos ${ }^{[2]}$.

As fibras lignocelulósicas possuem três componentes principais: hemicelulose (20 a 40\% em massa), celulose (40 a $60 \%$ em massa) e lignina (10 a $25 \%$ em massa). A celulose que é o componente majoritário é um polissacarídeo semicristalino que contém ligações $\beta$ glicosídicas. A presença de três grupos hidroxila em cada unidade repetitiva da celulose resulta em um caráter hidrofílico $^{[3]}$. As fibras lignocelulósicas e as poliolefinas possuem caráter hidrofílico e hidrofóbico, respectivamente,

Autor para correspondência: Marco-A. De Paoli, Laboratório de Polímeros Condutores e Reciclagem, Instituto de Química,

Universidade Estadual de Campinas, CP 6154, CEP 13084-970, Campinas, SP, Brasil. E-mail: mdepaoli@iqm.unicamp.br 
resultando em regiões de aglomeração além da transferência da tensão ser prejudicada pela fraca adesão nas interfaces polímero/ fibra $^{[3]}$. A fim de aumentar a adesão entre estas fases é necessário o uso de agentes de acoplagem, como por exemplo, o polipropileno enxertado com anidrido maleico ou com ácido acrílico.

Várias fibras vegetais vêm sendo usadas em compósitos com polímeros, tais como: rami, fibra de coco, algodão, juta, sisal e curaua ${ }^{[2]}$. Dentre as fibras lignocelulósicas com potencial de aplicação na área de polímeros reforçados destaca-se a fibra extraída das folhas do curauá. Ela tem recebido atenção especial dos pesquisadores, pois possui propriedades mecânicas adequadas para o reforço em relação às outras fibras vegetais. O curauá é uma planta da família das bromeliáceas (Ananas erectifolius L.B. Smith), cujo teor médio dos componentes é de $74 \%$ de Celulose, $10 \%$ de Hemicelulose, $84 \%$ de Holocelulose, $7 \%$ de Lignina e $1 \%$ de cinzas $^{[4]}$. Esses teores variam em função da espécie (branco ou roxo), do local de plantação e do tempo decorrido após a colheita ${ }^{[5]}$. As folhas chegam a $1,5 \mathrm{~m}$ de comprimento e $4 \mathrm{~cm}$ de largura, são duras, eretas e planas. Ele é cultivado na região Amazônica brasileira, particularmente no Estado do Pará.

Existem diversos relatos de preparação de compósitos com fibras de curauá utilizando termofixos ${ }^{[6]}$ e o nosso grupo de pesquisas estuda vários aspectos relacionados ao processamento por extrusão e injeção de compósitos de termoplásticos com a fibra de curaua ${ }^{[4,7]}$. Os compósitos obtidos por estes processos podem ser utilizados para a confecção de perfis ou produtos acabados com forma complexa usando moldagem por injeção. No desenvolvimento dos trabalhos foi verificado que o teor de fibra de curauá que proporciona as melhores propriedades mecânicas (tração, flexão e impacto) aos compósitos de polipropileno ou polietileno de alta densidade corresponde à $20 \%$ em massa de fibra e $2 \%$ em massa de polipropileno ou polietileno modificado com anidrido maleico, que atuam como agente de acoplagem.

Outra fibra importante é a fibra de sisal, devido ao fato de corresponder a cerca de $70 \%$ da produção comercial brasileira de todas as fibras duras. A produção anual brasileira de sisal é da ordem de 270.000 t/ano e o cultivo se concentra na região Nordeste, sendo o estado da Bahia o principal produtor ${ }^{[8]}$. Martin et al. ${ }^{[8]}$ caracterizaram o teor dos constituintes principais da fibra de sisal e relataram a seguinte composição: 77 a $85 \%$ de celulose, 7 a $10 \%$ de hemicelulose, 7 a $11 \%$ de lignina e 1 a 1,5\% de cinzas. Também existem muitos relatos de preparação de compósitos poliméricos com fibras de sisal ${ }^{[9-14]}$.

Apesar do grande número de trabalhos já existente, não é possível comparar as propriedades finais dos compósitos relatadas, porque variam os métodos de processamento, o tipo e teor de agentes de acoplagem e o teor e dimensões das fibras, Há então uma grande controvérsia quanto às propriedades finais dos compósitos destas duas fibras com polietileno de alta densidade, PEAD, ou com polipropileno, PP. Em compósitos a forma de dispersão do agente de reforço, as dimensões do mesmo e o agente de acoplagem influenciam muito nas propriedades mecânicas finais ${ }^{[15]}$. Por outro lado, a forma de dispersão ou forma de processamento também vai definir as dimensões finais da fibra, refletidas na sua razão de aspecto ${ }^{[16]}$. Portanto, julgamos importante comparar as propriedades mecânicas de compósitos de PP ou PEAD, preparados com $20 \%$ em massa de fibras de sisal ou de curauá e $2 \%$ em massa de agente de acoplagem, usando o mesmo equipamento de extrusão (extrusora dupla-rosca co-rotante e interpenetrante), o mesmo perfil de rosca e os mesmos perfis de temperatura. Com esses resultados poderemos definir com melhor precisão em quais aplicações cada uma destas fibras será mais adequada.

\section{Experimental}

\section{Materiais}

Utilizou-se o polipropileno homopolímero (PP H301, MFI = $10 \mathrm{~g} / 10$ minutos) e o polietileno de alta densidade (PEAD JV 060U, MFI = 6,1-8,0 g / 10 minutos) na forma de pellets, ambos fornecidos pela Braskem. Foram usados os agentes de acoplagem: poli(propileno-co-anidrido maleico) e poli(etileno-co-anidrido maleico) comerciais (PP-g-AM, MEGHWAX SGW X-01, índice de acidez, mg KOH/g = 37-50 e PE-g-AM, MEGHWAX SAW X-02 índice de acidez, mg KOH/g = 100-130) fornecidos pela Megh Indústria \& Comércio Ltda. As fibras de curauá na forma de fibras longas após colheita e extração seguida de secagem foram fornecidas pela EMBRAPA - PA.

\section{Metodologia}

\section{Caracterização das fibras de sisal e curaua}

As fibras foram moídas em moinho de facas (Rone, modelo NFA 1533) e peneiradas. Para a determinação da distribuição da freqüência de dimensões das fibras de curaua e sisal, as fibras moídas foram dispostas em placas de vidro de modo a não ficarem sobrepostas, foram observadas em uma lupa (Micronal) e fotografadas com uma câmera (Nikon E-800) acoplada ao sistema de captura de imagens do software Image Pro Plus ${ }^{\circledR}$. As imagens digitalizadas foram analisadas, sendo realizada a contagem do número e do comprimento das fibras. $\mathrm{O}$ espaço amostral utilizado foi de cerca de 400 fibras e o tratamento estatístico dos dados foi realizado com o software Origin ${ }^{\circledR}$.

Foram realizados ensaios de densidade em duplicata das fibras moídas previamente secas $\left(100{ }^{\circ} \mathrm{C}, 1\right.$ hora $)$ usando o equipamento Micromeritics modelo AccuPyc II 1340 V1.02.

Os ensaios de tração das fibras foram realizados seguindo a norma ASTM D3379 usando o equipamento universal de ensaios EMIC modelo DL 2000 (célula de carga de $500 \mathrm{~N}$ e velocidade de $2,0 \mathrm{~mm} / \mathrm{min}$ ). Antes das medidas as fibras foram condicionadas a $25{ }^{\circ} \mathrm{C}$ e $50 \%$ de umidade relativa durante 24 horas. Esse procedimento é importante porque a umidade pode atuar como plastificante das fibras.

Foram realizadas medidas de microscopia eletrônica de Varredura da superfície das fibras usando o equipamento JEOL JSM-6360LV.

\section{Processamento dos compósitos}

Os homopolímeros, as fibras moídas e os compatibilizantes foram previamente misturados por tombamento por 2 minutos e secos por 1,5 horas a $100{ }^{\circ} \mathrm{C}$ em estufa. Os compósitos foram processados na extrusora dupla-rosca co-rotante interpenetrante (ZSK $26 \mathrm{Mc}$, Coperion Werner \& Pfleiderer, L/D = 44) a $300 \mathrm{rpm}$. As fibras foram alimentadas com um dosador e usando um alimentador lateral com rotação de roscas de $265 \mathrm{rpm}$ e foi utilizada a degasagem sob vácuo durante toda a duração do processamento. O torque variou de 25 a $35 \%$ durante o processo. O perfil de temperatura usado foi de $160,160,165,165,170,170,170,170,180,175,170{ }^{\circ} \mathrm{C}$ para o PP e $120,120,125,125,130,130,135,135,140,135,130{ }^{\circ} \mathrm{C}$ para o PEAD. Os compósitos foram resfriados com água e picotados de forma contínua. Os pellets foram secos por 1 hora a $100{ }^{\circ} \mathrm{C}$ e injetados (Arburg, All Rounder M-250) na forma de corpos de prova para ensaios mecânicos de tração (ASTM D 638-02, Tipo I), flexão (ASTM D 790) e impacto (ASTM D 256), com perfil de temperatura de 185 a $195{ }^{\circ} \mathrm{C}$ e de 130 a $160{ }^{\circ} \mathrm{C}$ da alimentação até o bico de injeção, para o PP e PEAD, respectivamente. As pressões de injeção foram 1300-1400 bar e recalque 750-800 bar, velocidade 
de injeção de $15 \mathrm{~cm}^{3} \cdot \mathrm{s}^{-1}$, temperatura do molde de $35^{\circ} \mathrm{C}$ e tempo de resfriamento do molde de 10 segundos.

\section{Caracterização dos compósitos}

\section{Ensaios de resistência à tração, à flexão e ao impacto}

Os ensaios de tração e flexão foram realizados de acordo com as normas ASTM D638 e D790, respectivamente, usando velocidades de separação das garras nos ensaios de tração e flexão de 50 e $5,3 \mathrm{~mm} / \mathrm{min}$, respectivamente. Os ensaios de resistência ao impacto foram realizados de acordo com a norma ASTM D 256, método A, usando martelo com energia de 5,54 J. As propriedades foram avaliadas considerando-se os resultados obtidos para 10 corpos de prova com entalhe.

\section{Microscopia eletrônica de varredura (MEV)}

Os corpos de prova injetados foram crio-fraturados após 15 minutos de permanência em $\mathrm{N}_{2}$ líquido. Sobre a superfície fraturada depositou-se uma película de ouro e paládio (razão 8:2) através de metalizador (Bal-Tec, Mult Coating System MED020). As micrografias foram realizadas com $20 \mathrm{kV}$ de aceleração usando um microscópio eletrônico de varredura (JEOL JSM-6360LV).

Determinação das dimensões das fibras após o processamento.

Para determinar as dimensões das fibras após o processamento dos compósitos (extrusão e injeção), 0,5 g de compósito foram extraídos com $50 \mathrm{~mL}$ de xileno a $130-135{ }^{\circ} \mathrm{C}$ durante 1 hora com agitação, ou até a completa dissolução da matriz. As fibras foram separadas por filtração, lavadas com três porções de xileno a quente e o solvente incorporado à fibra foi evaporado a $90{ }^{\circ} \mathrm{C}$. Para a determinação da distribuição da freqüência de dimensões das fibras usou-se o mesmo método utilizado para as fibras moídas.

\section{Resultados e Discussão}

\section{Propriedades das fibras}

As densidades medidas para as fibras de curauá e de sisal usadas nesse trabalho foram de $1100( \pm 91)$ e $1588( \pm 11) \mathrm{kg} \cdot \mathrm{m}^{-3}$, respectivamente. Esses valores são comparáveis à outras fibras vegetais como a fibra de juta $\left(1300 \mathrm{~kg} \cdot \mathrm{m}^{-3}\right)$, de coco $\left(1150 \mathrm{~kg} \cdot \mathrm{m}^{-3}\right)$, de algodão (1500-1600 kg.m $\left.{ }^{-3}\right)$ e linho $\left(1500 \mathrm{~kg} \cdot \mathrm{m}^{-3}\right)^{[17]}$. Como ilustrado nas micrografias apresentadas na Figura 1, as fibras de sisal e de curauá antes da moagem e do processamento apresentam diâmetro médio $\left(\varnothing_{\mathrm{m}}\right)$ de 235 e $65 \mu \mathrm{m}$, respectivamente. Pode-se observar também nas micrografias que as fibras são compostas por feixes de microfibrilas. As fibras de curauá têm um diâmetro quatro vezes menor que as fibras de sisal. Este aspecto, além do ângulo que as fibrilas se encontram na estrutura da fibra reflete nas suas propriedades mecânicas pois, de acordo com M. Hughes ${ }^{[18]}$, o módulo elástico das fibras vegetais diminui em função do aumento do ângulo das microfibrilas.

Os valores de tensão na força máxima $\sigma(\mathrm{MPa})$, módulo de elasticidade $\mathrm{E}(\mathrm{GPa})$ e alongamento na ruptura $\varepsilon(\%)$ para as fibras de curauá e sisal são mostrados na Tabela 1 . O valor de tensão na força máxima da fibra de curauá é quase o dobro do valor para a fibra de sisal. O módulo de elasticidade da fibra de curauá é ligeiramente maior e o alongamento na ruptura para as duas fibras é praticamente idêntico. Em termos das propriedades mecânicas, é a tensão na força máxima que deverá ter a maior influência nas propriedades de reforço da fibra. Esses resultados são comparáveis porque as medidas para as duas fibras foram feitas exatamente da mesma maneira e representam uma média de 100 amostras.

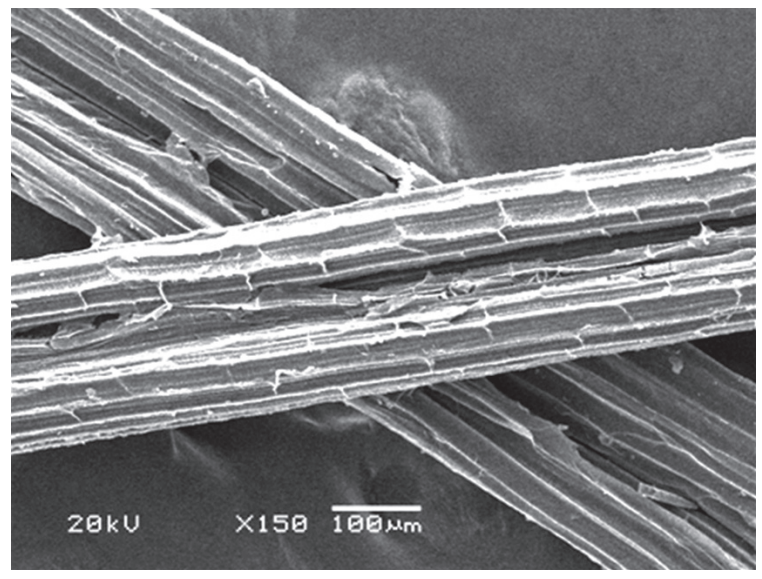

(a)

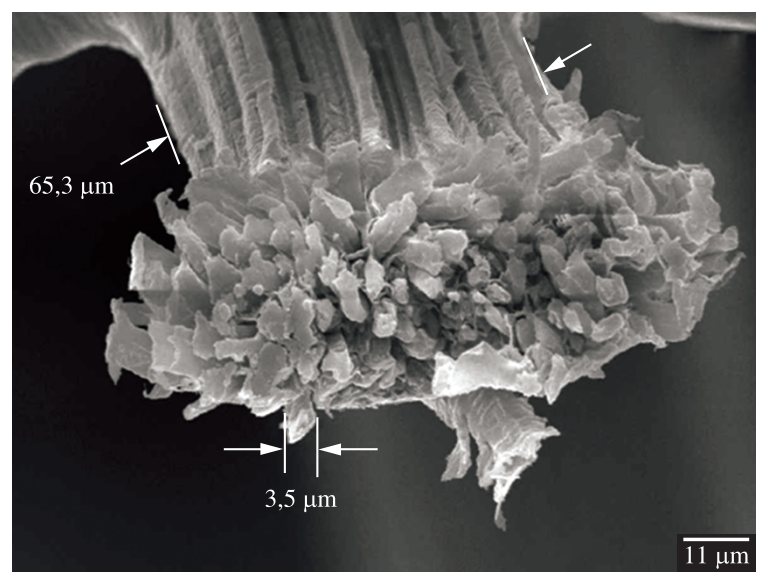

(b)

Figura 1. Micrografias obtidas por Microscopia Eletrônica de Varredura das fibras: a) sisal; e b) curauá.

Tabela 1. Valores de tensão na força máxima $(\sigma)$, módulo de elasticidade $(E)$ e alongamento na ruptura $(\varepsilon)$ para as fibras de sisal e curauá (média de 100 amostras).

\begin{tabular}{lccc}
\hline & $\sigma / \mathbf{M P a}$ & $\mathbf{E} / \mathbf{~ G P a}$ & $\varepsilon / \%$ \\
\hline Sisal & $264( \pm 72)$ & $10,5( \pm 0,6)$ & $4,0( \pm 1,5)$ \\
Curauá & $509( \pm 109)$ & $19( \pm 4)$ & $3,0( \pm 0,3)$ \\
\hline
\end{tabular}

\section{Propriedades dos compósitos}

Os gráficos a e b da Figura 2 mostram as distribuições do comprimento médio das fibras após a moagem. A razão entre comprimento e o diâmetro das fibras (razão de aspecto ou L/ $\varnothing_{\mathrm{m}}$ ), considerando o comprimento onde temos a maior porcentagem de fibras e o diâmetro médio determinado nas micrografias, é de 167 para o curauá $\left(\mathrm{L}=10 \mathrm{~mm}\right.$ e $\left.\varnothing_{\mathrm{m}}=60 \mu \mathrm{m}\right)$ e 5,3 para o sisal $\left(\mathrm{L}=1,25 \mathrm{~mm}\right.$ e $\left.\varnothing_{\mathrm{m}}=235 \mu \mathrm{m}\right)$. A maior diminuição do comprimento da fibra de sisal em relação ao curauá é uma indicação de maior fragilidade, uma vez que foram utilizadas exatamente as mesmas condições de moagem. A maior redução do comprimento leva a um valor menor de razão de aspecto para as fibras de sisal moídas.

Os gráficos c, d, e, f da Figura 2 mostram a distribuição de tamanho das fibras extraídas dos corpos de prova obtidos por injeção dos compósitos com matrizes de PEAD e PP. Após o processamento dos compósitos na extrusora dupla-rosca notamos que houve uma maior redução de comprimento para as fibras 


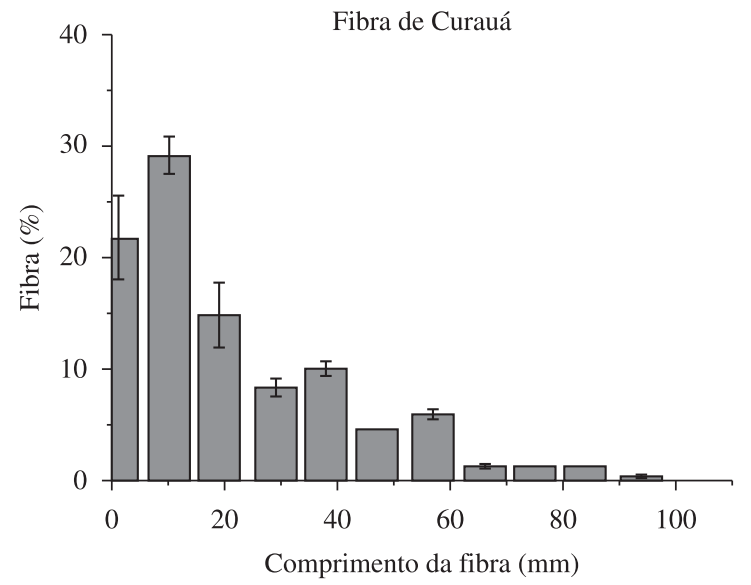

(a)

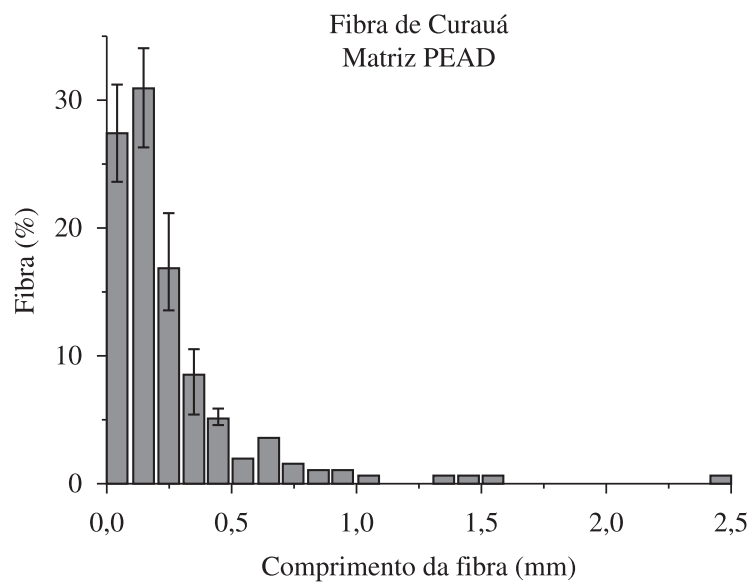

(c)

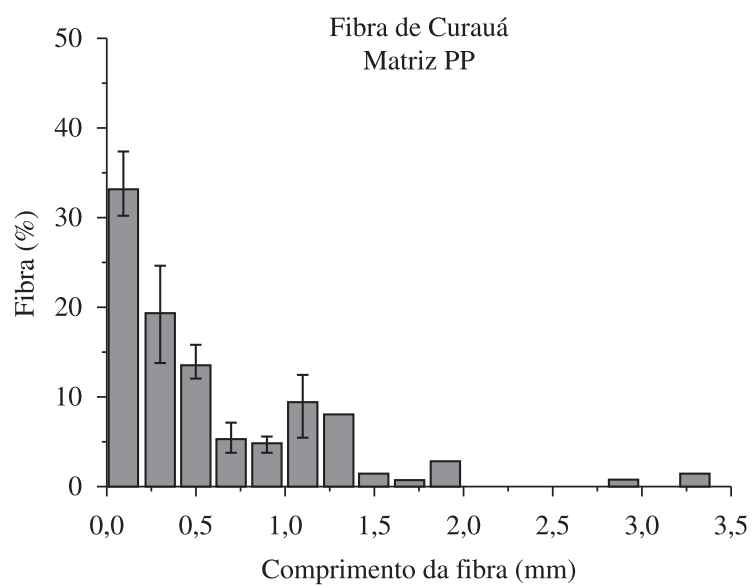

(e)

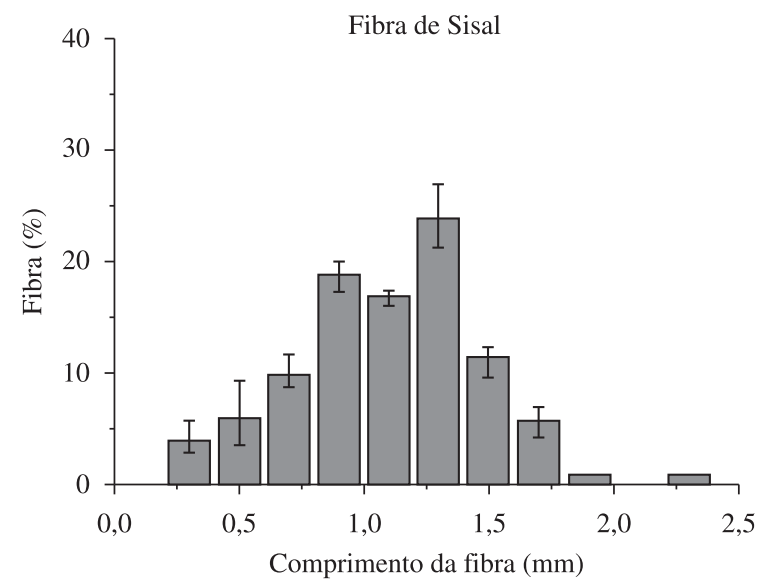

(b)

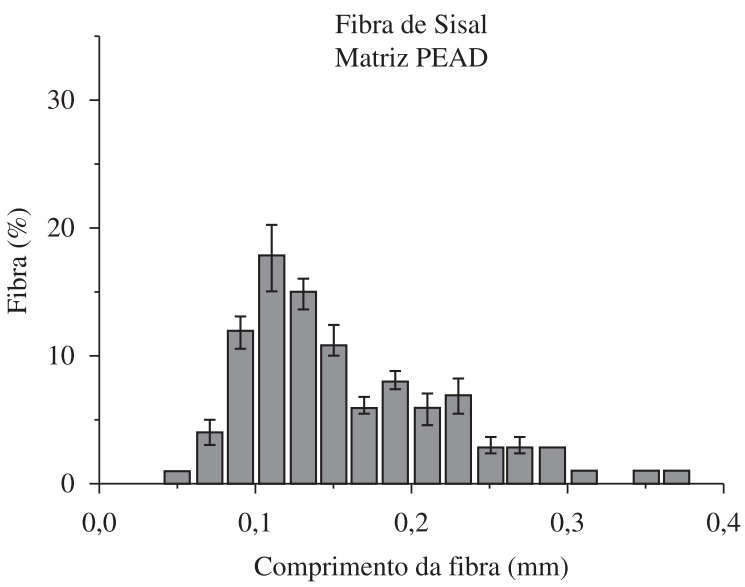

(d)

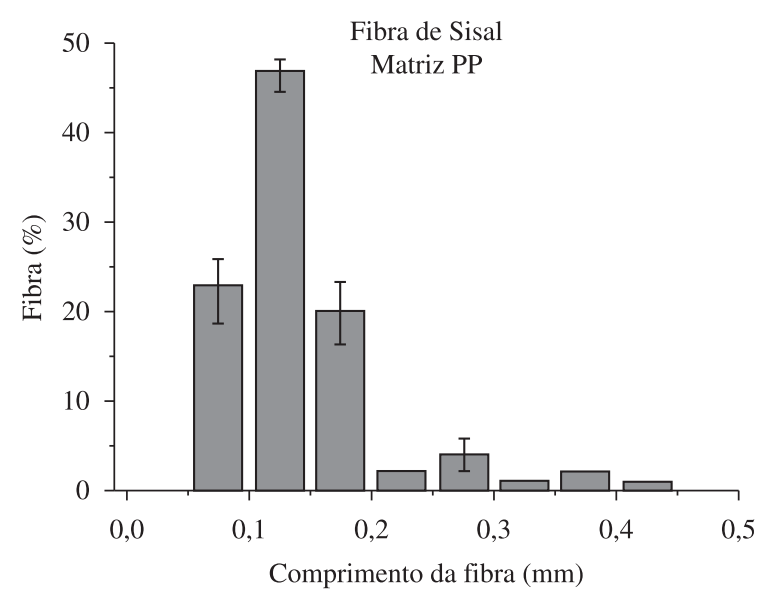

(f)

Figura 2. Distribuição de comprimento das fibras de curauá e sisal depois da moagem (a, b) e depois do processamento com matrizes de PEAD (c, d) e PP $(\mathrm{e}, \mathrm{f})$.

de curauá (Figuras 2c e 2e) do que de sisal (Figuras 2d e 2f). A redução de tamanho (e de diâmetro) é causada pelo cisalhamento no processamento, mas aparentemente a redução de tamanho também é afetada pela fase continua utilizada. No caso dos compósitos usando as fibras de curauá a redução de comprimento foi maior para os compósitos com matrizes de PEAD, Figuras 2a, c e e. Já para os compósitos usando o sisal como reforço a redução é similar para as duas matrizes usadas, Figura 2b, d e f. Se considerarmos que nos dois casos houve fibrilação reduzindo também o diâmetro das fibras, as propriedades mecânicas finais serão controladas pela razão de aspecto das fibras após o processamento.

Na Tabela 2 mostramos as propriedades mecânicas em ensaios de tração, flexão e impacto para os corpos de prova injetados com os compósitos processados com sisal ou curauá usando PP ou PEAD como matrizes. Para os ensaios de tração e flexão, observa-se que as propriedades mecânicas dos corpos de prova dos compósitos usando as fibras de sisal como reforço, tanto com a matriz de PP como a matriz de PEAD, são inferiores quando comparadas com 
Spinacé, M. A. S. et al. - Poliolefinas reforçadas com fibras vegetais curtas: sisal vs. curauá

Tabela 2. Propriedades mecânicas dos compósitos de PP e PEAD com fibras de sisal e de curauá, $\sigma=$ tensão na força máxima, $\varepsilon=$ deformação na ruptura e E módulo.

\begin{tabular}{|c|c|c|c|c|}
\hline & \multicolumn{2}{|c|}{ PP/fibra (80/20) } & \multicolumn{2}{|c|}{ PP/fibra/PP-g-AM (78/20/2) } \\
\hline & \multicolumn{4}{|c|}{ Tração } \\
\hline & Curauá* & Sisal & Curauá* & Sisal \\
\hline$\sigma(\mathrm{MPa})$ & $31,58( \pm 0,55)$ & $28,62( \pm 0,13)$ & $30,90( \pm 0,20)$ & $24,87( \pm 0,57)$ \\
\hline$\varepsilon(\%)$ & $4,04( \pm 0,25)$ & $5,25( \pm 0,51)$ & $4,41( \pm 0,43)$ & $6,15( \pm 1,36)$ \\
\hline \multirow[t]{2}{*}{$\mathrm{E}(\mathrm{GPa})$} & $3,15( \pm 0,35)$ & $2,81(0,52)$ & $2,69( \pm 0,34)$ & $2,75( \pm 0,27)$ \\
\hline & \multicolumn{4}{|c|}{ Flexão } \\
\hline$\sigma(\mathrm{MPa})$ & $53,62( \pm 0,28)$ & $49,74( \pm 1,39)$ & $51,92( \pm 0,27)$ & $40,38( \pm 1,54)$ \\
\hline \multirow[t]{5}{*}{$\mathrm{E}(\mathrm{GPa})$} & $2,25( \pm 0,05)$ & $2,17( \pm 0,05)$ & $2,12( \pm 0,08)$ & $1,89( \pm 0,07)$ \\
\hline & \multicolumn{4}{|c|}{ Resistência ao impacto $\left(\mathrm{J}^{\prime} \mathrm{m}^{-1}\right)$} \\
\hline & $31,3( \pm 2,8)$ & $34,60( \pm 2,56)$ & $21,9( \pm 0,6)$ & $36,5( \pm 3,97)$ \\
\hline & \multicolumn{2}{|c|}{ PEAD/fibra (80/20) } & \multicolumn{2}{|c|}{ PEAD/fibra/PE-g-AM(78/20/2) } \\
\hline & \multicolumn{4}{|c|}{ Tração } \\
\hline$\sigma(\mathrm{MPa})$ & $29,70( \pm 0,34)$ & $22,71( \pm 0,10)$ & $30,38( \pm 0,48)$ & $22,71( \pm 0,05)$ \\
\hline$\varepsilon(\%)$ & $2,9( \pm 0,31)$ & $7,52( \pm 0,91)$ & $3,00( \pm 0,29)$ & $7,09( \pm 1,08)$ \\
\hline \multirow[t]{2}{*}{$\mathrm{E}(\mathrm{GPa})$} & $2,83( \pm 0,58)$ & $1,99( \pm 0,23)$ & $2,45( \pm 0,26)$ & $2,33( \pm 0,49)$ \\
\hline & \multicolumn{4}{|c|}{ Flexão } \\
\hline$\sigma(\mathrm{MPa})$ & $40,42( \pm 0,95)$ & $35,27( \pm 1,02)$ & $40,92( \pm 0,27)$ & $35,30( \pm 0,94)$ \\
\hline \multirow[t]{3}{*}{$\mathrm{E}(\mathrm{GPa})$} & $1,89( \pm 0,12)$ & $1,79( \pm 0,31)$ & $1,96( \pm 0,13)$ & $2,03( \pm 0,13)$ \\
\hline & \multicolumn{4}{|c|}{ Resistência ao impacto $\left(\mathrm{J}^{\prime} \mathrm{m}^{-1}\right)$} \\
\hline & $62,05( \pm 2,91)$ & $65,5( \pm 2,37)$ & $65,17( \pm 3,93)$ & $67,5( \pm 3,20)$ \\
\hline
\end{tabular}

* Os resultados referentes aos compósitos com fibra de curauá foram extraídos das referências 19 e 20.

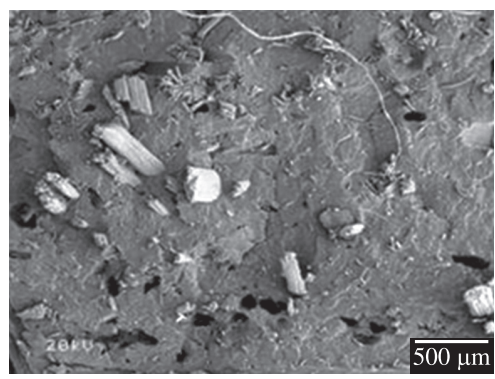

(a)

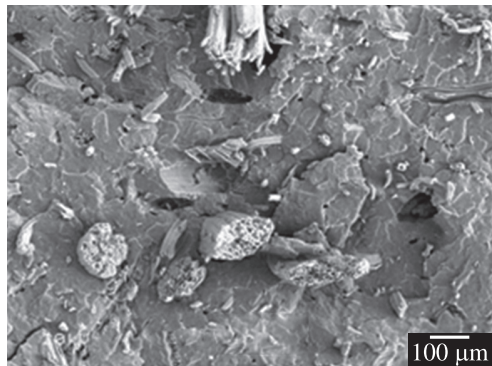

(d)

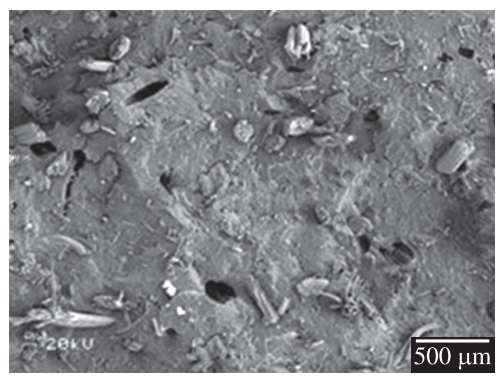

(b)

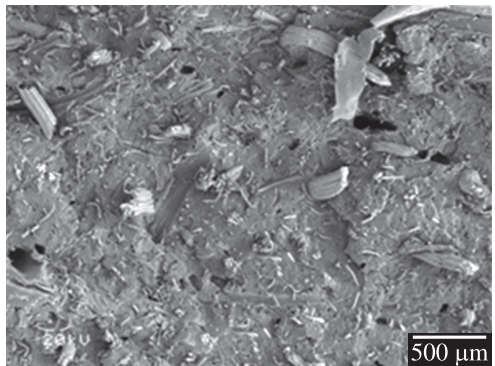

(e)

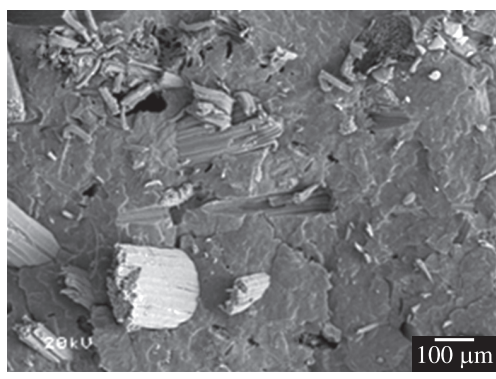

(c)

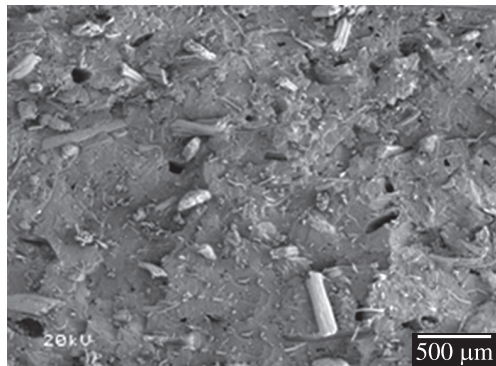

(f)

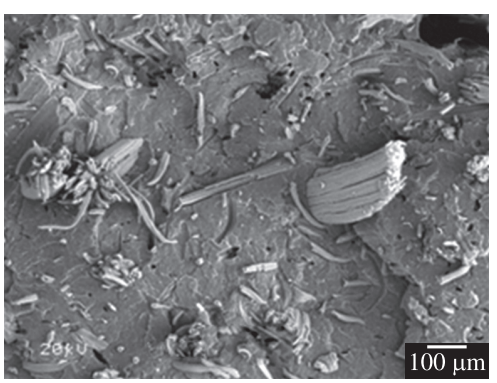

(g)

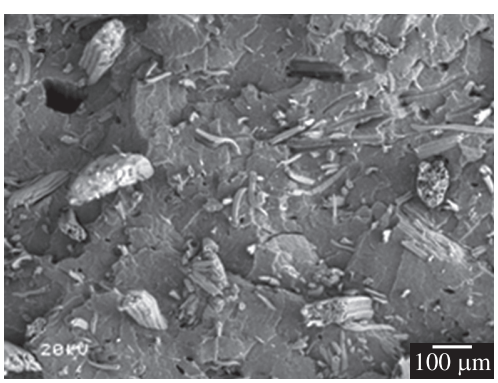

(h)

Figura 3. Micrografias obtidas por Microscopia Eletrônica de Varredura dos compósitos com fibra de sisal: a) 80 PP/20 fibra de sisal; b) 78 PP/20 fibra de sisal/2PP-g-AM; c) 80 PP/20 fibra de sisal; d) 78 PP/20 fibra de sisal/2PP-g-AM; e) 80 PEAD/20 fibra de sisal; f) 78 PEAD/20 fibra de sisal/2PE-g-AM; g) 80 PEAD/20 fibra de sisal; e h) 78 PEAD/20 fibra de sisal/2PE-g-AM. 


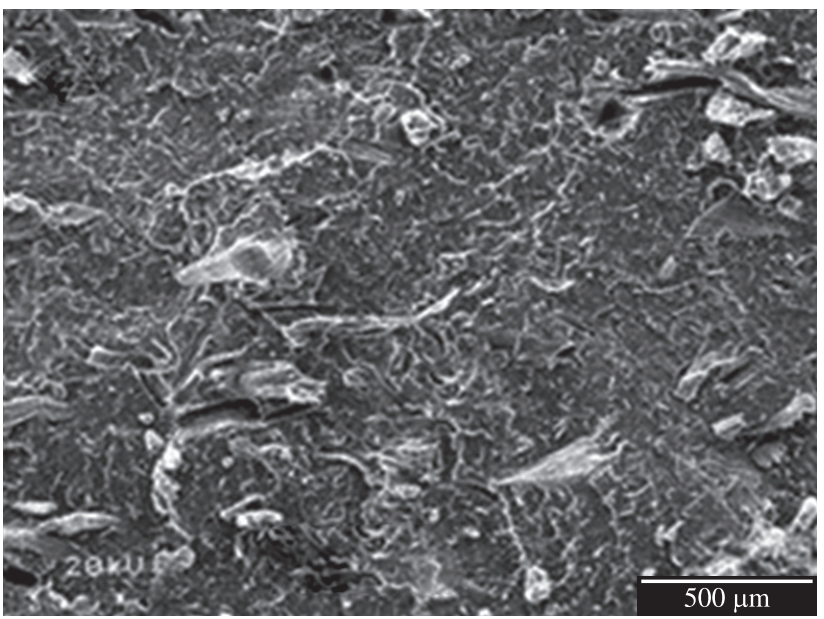

(a)

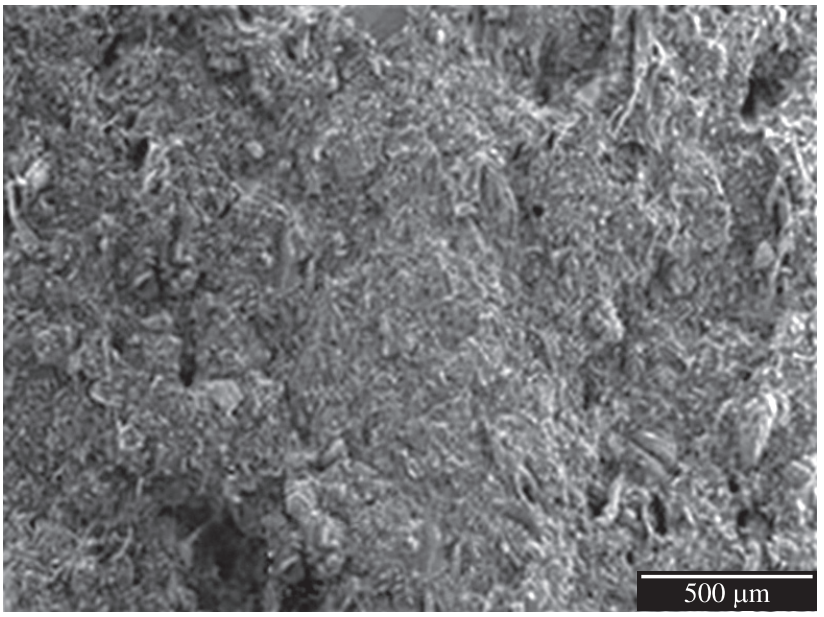

(c)

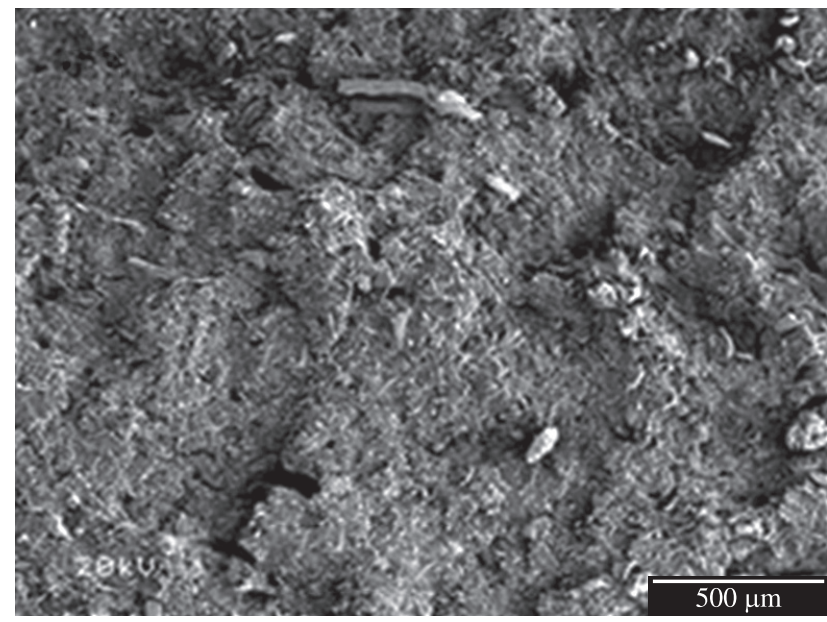

(b)

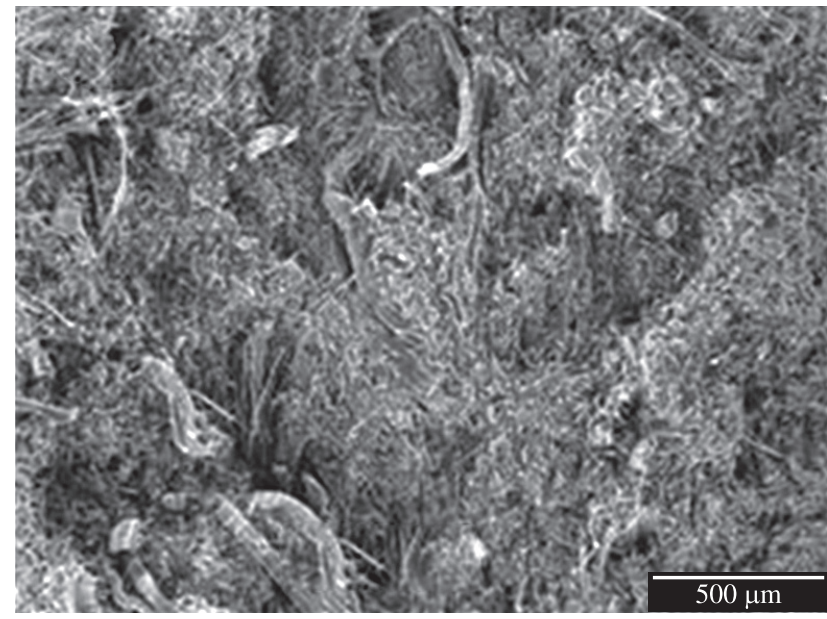

(d)

Figura 4. Micrografias obtidas por Microscopia Eletrônica de Varredura dos compósitos obtidos com fibra de curauá: a) 80 PP/20 fibra de curauá; b) 78 PP/20 fibra de curauá/2PP-g-AM; c) 80 PEAD/20 fibra de curauá; e d) 78 PEAD/20 fibra de curauá/2PE-g-AM.

os compósitos usando as fibras de curauá. Esta diferença ocorre tanto na presença como na ausência de agente de acoplagem para as duas matrizes. Este resultado era esperado uma vez que as fibras de sisal apresentam menor resistência à tração do que o curauá. Além disso, como as fibras de sisal são mais frágeis, estas quebraram mais durante a moagem, resultando em razão L/D muito menor ( 5) o que não favorece uma transferência eficiente de tensão entre a matriz e a fibra. No caso da resistência ao impacto, observamos que os compósitos com sisal apresentaram valores ligeiramente superiores aos compósitos com curauá.

Analisando-se os resultados da Tabela 2, observa-se ainda que, tanto no caso da fibra de curauá como de sisal, e para as duas matrizes (PEAD e PP), o uso do agente de acoplagem não afeta de maneira acentuada as propriedades mecânicas. Os resultados referentes aos compósitos com fibra de curauá foram extraídos das referências 19 e 20.

No caso do compósito de PP com fibra de sisal o uso do agente de acoplagem ainda leva a uma diminuição da resistência do compósito. Quando a matriz é PEAD não ocorre variação significativa nas propriedades mecânicas. Esses resultados indicam que, para as fibras de sisal e curauá o agente de acoplagem utilizado não promove uma melhora eficiente das propriedades mecânicas. Será necessário um estudo mais detalhado do processo de acoplagem para se chegar ao sistema mais adequado.
As micrografias por MEV da seção de fratura dos compósitos, Figura 3, demonstram que o método de processamento proporciona uma boa dispersão das fibras nas matrizes. Além disso, observa-se que, paralelamente à diminuição do comprimento das fibras, também ocorre diminuição do seu diâmetro devido ao efeito de fibrilação durante o processamento. Esse efeito já foi observado anteriormente para a fibra de curauá ${ }^{[19]}$. No presente trabalho observa-se que a fibrilação ocorre para os compósitos com fibras de curauá e de sisal como pode ser verificado na Figura 3. As micrografias refletem o comportamento mecânico, pois não é possível verificar diferença significativa na morfologia dos compósitos com e sem agente de acoplagem, Figura 4.

\section{Conclusões}

Os compósitos de polietileno de alta densidade e polipropileno reforçados com fibras de curauá apresentam propriedades de resistência mecânica no modo tração e flexão, superiores quando comparadas aos compósitos com fibras de sisal usando as mesmas matrizes e processados nas mesmas condições. Para os ensaios de resistência ao impacto não há uma diferença significativa entre os compósitos preparados com as duas fibras. Estes resultados estão relacionados às propriedades mecânicas das fibras de sisal comparadas com as das fibras de curauá. A fragilidade da fibra 
de sisal provoca a quebra da mesma no processo de moagem, reduzindo a sua razão de aspecto e influenciando a eficiência de reforço no compósito. Estes resultados indicam que é possível obter compósitos com características e aplicações distintas, selecionando o método de processamento, a poliolefina e a fibra adequada.

\section{Agradecimentos}

Os autores agradecem a FAPESP pelo auxílio financeiro (2004/15084-6) e pelas bolsas de apoio técnico para LGJ (06/54835-2), FCB (08/06503-6) e TG (08/06566-8). M. A. De Paoli agradece a bolsa de produtividade do CNPq. Os autores agradecem a Braskem pelas amostras de poliolefinas, a MEGH pelos agentes de acoplagem e a Embrapa-PA pelas fibras.

\section{Referências Bibliográficas}

1. Abiplast. Disponível em: <www.abiplast.org.br>. Acesso em: 13 abr. 2010.

2. Gassan, A. K. \& Bledski, J. - Prog. Polym. Sci., 24, p.221 (1999).

3. Spinacé, M. A. S.; Lamber, C. S.; Fermoselli, K. K. G. \& De Paoli, M. A. - Carbohydr. Polym., 77, p.47 (2009).

4. Santos, P. A.; Spinacé, M. A. S.; Fermoselli, K. K. G. \& De Paoli, M. A. - Polímeros, 19, p.31 (2009).

5. Behrens, D. - "Curauá-Fäser - eine Pflanzenfaser als Konstruktionswerkstof ?", Verlag Dr. Köster, Berlin, p.159 (1999).

6. Trindade, W. G.; Hoareau, W.; Megiatto, J. D.; Razera, I. A. T.; Castellan, A. and Frollini, E. - Biomacromology, 6, p.2485 (2005).

7. Spinacé, M. A. S.; Fermoselli, K. K. G. \& De Paoli, M. A. - J. Appl. Polym. Sci. 112, p.3686 (2009).

8. Martin, A. R.; Martins, M. A.; Mattoso, L. H. C. \& Silva, O. R. R. F. Polímeros, 19, p.40 (2009).
9. Iozzi, M. A.; Martins, G. S.; Martins, M. A.; Ferreira, F. C.; Job, A. E. \& Mattoso, L. H. C. - Polímeros, 20, p.25 (2010).

10. Carvalho, L. H. \& Cavalcanti, W. S. - Polímeros, 16, p.33 (2006).

11. Martins, G. S.; Iozzi. M. A.; Martins. M. A.; Mattoso. L. H. C. \& Ferreira F. C. - Polímeros, 14, p.326 (2004).

12. Iannace, S.; Ali, R. \& Nicolais, L. - J. Appl. Polym. Sci., 79, p.1084 (2001)

13. Joseph, P. V.; Joseph, K. \& Thomas, S. - Compos. Sci. Technol., 59, p.1625 (1999).

14. Josepha, P. V.; Rabello, M. S.; Mattoso, L. H. C.; Josepha, K. \& Thomas, S. - Compos. Sci. Technol., 62, p.1357 (2002).

15. Rabello, M. - "Aditivação de Polimeros", Artliber, São Paulo, p. 193 (2000).

16. Mano, B.; Araújo, J. R.; Spinacé, M. A. S. \& De Paoli, M-A. - Compos. Sci. Technol., 70, p.29 (2010).

17. Marinelli, A. L.; Monteiro, M. R.; Ambrosio, J. D.; Branciforti, M. C.; Kobayashi, M. \& Nobre, A. D. - Polimeros, 18, p.92 (2008).

18. Hughes, M. - in: "Low Environmental Impact Polymers", N. Tucker \& M. Johnson (Eds)., Rapra Technology, Shawbury, p. 80 (2004).

19. Araujo, J. R.; Mano, B. I. S.; Spinacé, M. A. S. \& De Paoli, M.-A. "Vegetal biomicrofibril polyolefin composites prepared by extrusion", in: "The Polymer Processing Society 24 $4^{\text {th }}$ Annual Meeting", Salerno, p.1-6 (2008).

20. Mano, B. I.; Spinacé, M. A. S. \& De Paoli, M. A. - "Polypropylene composite reinforced with natural fiber: processing and coupling agent effect" in: "Anais 23 $3^{\text {th }}$ Annual Meeting of the Polymer Processing Society", Salvador, BA, p.3-17 (2007).

Enviado: 15/04/10

Reenviado: 20/09/10

Aceito: $23 / 10 / 10$

DOI: 10.1590/S0104-14282011005000036 